\title{
Therapeutic effects and associated adverse events of multikinase inhibitors in metastatic renal cell carcinoma: A meta-analysis
}

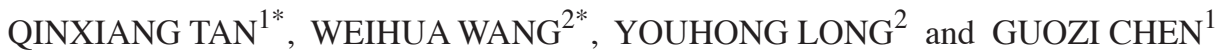 \\ ${ }^{1}$ Nephrology Department, Longgang Hospital of Traditional Chinese Medicine, Shenzhen, Guangdong 518000; \\ ${ }^{2}$ Nephrology Department, Guangzhou Hospital of Integrated Traditional and Western Medicine, \\ Guangzhou, Guangdong 510000, P.R. China
}

Received June 23, 2014; Accepted March 11, 2015

DOI: $10.3892 /$ etm.2015.2427

\begin{abstract}
This study aimed to compare the therapeutic effects and adverse events of the multikinase inhibitors sorafenib, sunitinib, pazopanib and axitinib in advanced renal cell carcinoma (RCC). A meta-analysis of randomized controlled trials was performed to assess the effects of multikinase inhibitors among patients with advanced RCC. The data of median progression-free survival (PFS), median overall survival (OS), progressive disease rate (PDR), objective response rate (ORR) and grade 3/4 adverse events were extracted to assess therapeutic effects and toxicity, respectively. It was found that multikinase inhibitors are more effective in extending PFS [hazard ratio $(\mathrm{HR})=0.58 ; 95 \%$ confidence interval $(\mathrm{CI})$ : 0.45-0.74; $\mathrm{P}<0.0001)$, controlling tumor progression [relative risk $(\mathrm{RR})=0.67 ; 95 \% \mathrm{CI}: 0.55-0.83 ; \mathrm{P}=0.0002)$ and $\mathrm{ORR}$ $(\mathrm{RR}=2.93 ; 95 \% \mathrm{CI}: 1.40-6.14 ; \mathrm{P}=0.004)$ compared with placebo or interferon- $\alpha$. Patients treated with multikinase inhibitors had significantly higher rates of grade 3 or 4 hypertension ( $\mathrm{RR}=6.00$; 95\% CI: 3.36-10.69; $\mathrm{P}<0.00001)$, diarrhea ( $R R=5.84 ; 95 \%$ CI: 3.06-11.16; $P<0.00001)$, nausea $(\mathrm{RR}=2.30$; 95\% CI: 1.16-4.54; $\mathrm{P}=0.02)$, vomiting $(\mathrm{RR}=1.84 ; 95 \% \mathrm{CI}$ : 1.00-3.41; $\mathrm{P}=0.05)$ and hand-foot skin reaction $(\mathrm{RR}=11.78$; 95\% CI: 5.16-26.93; P<0.00001). Multikinase inhibitors can significantly control disease progress and improve the ORR. However, they are also associated with a higher risk of grade 3 and 4 hypertension and gastrointestinal events. Proper management of these events is necessary to improve patient quality of life.
\end{abstract}

Correspondence to: Dr Youhong Long, Nephrology Department, Guangzhou Hospital of Integrated Traditional and Western Medicine, 87 Yinbin Road, Guangzhou, Guangdong 510000, P.R. China

E-mail: weihuawang1@outlook.com

${ }^{*}$ Contributed equally

Key words: multikinase inhibitors, renal cell carcinoma, meta-analysis

\section{Introduction}

Renal cell carcinoma (RCC) is one of the most progressive urological cancers among men and women $(1,2)$, and is considered as one of the most difficult cancers to diagnose and to treat (3). Advanced RCC is highly resistant to radiotherapy and chemotherapy. Approximately $25-30 \%$ of RCC patients suffer metastatic or advanced disease, with a 5-year survival rate $<10 \%$ (4). Prior to recent advancements in understanding of the molecular mechanism of RCC and the development of angiogenesis inhibitors, cytokine-based therapy such as interleukin-2 (IL-2) and interferon (IFN) was the main therapy used to treat RCC (5). However, the therapeutic effect of these treatments is quite limited, as there is only a 4-6\% combined response rate for most single agent or combination regimens (6).

Therapeutic options for patients have significantly expanded due to the successful development and adaptation of several targeting agents in the first-line treatment of advanced RCC, including multikinase inhibitors sorafenib (7), sunitinib (8), pazopanib (9) and axitinib (10); the combination of the anti-vascular endothelial growth factor (VEGF) agent bevacizumab with IFN- $\alpha$ (11); and mammalian target of rapamycin (mTOR) inhibitors temsirolimus (12) and everolimus (13). However, considering the poor physical condition, such as cachexia, that is common among patients with advanced RCC (14), selection of treatment should not only consider the therapeutic effect, but also adverse events. In order to make a more rational choice of treatment for individual patients with advanced RCC, it is necessary to identify the level of adverse effects of these options. In this scenario, a meta-analysis of randomized trials was performed to evaluate the therapeutic and adverse effects of the multikinase inhibitors sorafenib, sunitinib, pazopanib and axitinib.

\section{Materials and methods}

Study design. This study was a meta-analysis based on data collected from previous randomized controlled trials (RCTs) of first-line chemotherapies for patients with advanced RCC. Two reviewers (QT and YL) selected and reviewed the evidence independently. Disagreements were handled through group discussion. 
Search strategy. Studies were search among PubMed/Medline, Embase and Cochrane Central Register of Controlled Trials (CENTRAL) databases using the following terms and strategy: ('sorafenib' or 'sunitinib' or 'pazopanib' or 'axitinib') and ('renal cell carcinoma' or 'RCC' or 'metastatic renal cell carcinoma' or 'advanced RCC') and ('randomized trials' or 'random*' or 'RCT') in the abstract. The databases were searched for studies published up to February 2014. Only trials published in English were considered. Reference lists of related articles were manually checked to search for additional eligible publications. All references of relevant articles were scanned and all additional studies of potential interest were retrieved for further analysis.

Selection criteria. Eligible trials were required to meet the following criteria: Patients involved were diagnosed with advanced RCC through cytologic diagnosis or pathological diagnosis; RCTs evaluated multikinase inhibitors individually with a control intervention as the sole treatment; patients did not undergo surgery or other non-antiangiogenic treatment; and $\geq 100$ patients were enrolled. Animal studies, non-randomized trials and pharmacokinetic studies were excluded.

The bias risk of included publications was evaluated based on the Cochrane Handbook for Systematic Reviews of Interventions, version 5.0.0 (15). The major quality components include: i) Sequence generation of the allocation; ii) allocation concealment; iii) blinding of participants, personnel and outcome assessors; iv) incomplete outcome data; v) selective outcome reporting; and vi) other sources of bias (15). Trials were classified into three levels according to the bias risk. Trials with appropriate and sufficient support of index of outcome assessment that have minimal risk of bias were classified into level A; trials with one or more high or unclear risks for bias among the quality components and with a moderate level risk of bias were in level B; trials with three or more high or unclear risks for bias among the quality components and with the highest level of bias were in level C.

Data extraction. Two reviewers (QT and YL) separately and independently extracted data from the trials. Disagreements were handled by consensus. All data were checked for internal consistency. The trials were identified with the first author and the year of publication. For the trials that did not report the required data to determine the outcomes, the reviewers contacted the authors to obtain required information. The baseline information of patients and details of intervention of each trial were extracted to assess the heterogeneity. The outcomes assessed included tumor progression, objective response rate (ORR) and progressive disease rate (PDR). Toxicity data reported commonly in the trials involved were retrieved, extracted and assessed respectively.

Statistic analysis. Meta-analysis was conducted with Review Manager 5.2 (RevMan 5.2; The Nordic Cochrane Centre, The Cochrane Collaboration, Copenhagen, Denmark). Risk ratio (RR) was used for evaluation and the $95 \%$ confidence interval (CI) was calculated for each estimate. $\mathrm{P} \leq 0.05$ was used to denote statistical significance. Heterogeneity of the results of the trials was assessed with the $\mathrm{I}^{2}$ statistic using the $\chi^{2}$ test at $\alpha=0.1$ (15). Primary assessment was conducted with a fixed model. When $\mathrm{P} \geq 0.05$ and $\mathrm{I}^{2} \leq 50 \%$, it was considered

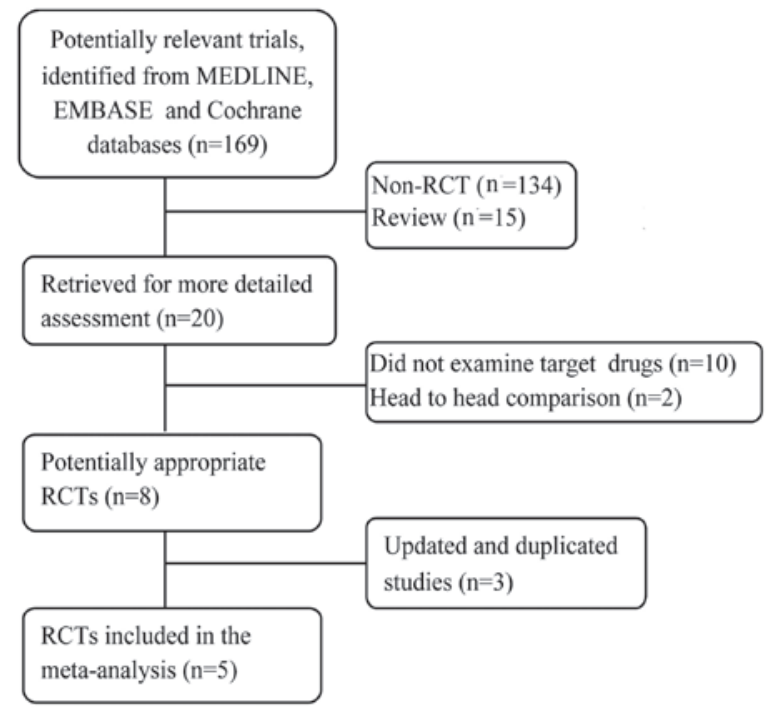

Figure 1. Flowchart of the literature search. RCT, randomized controlled trial.

that the trials were without heterogeneity and a fixed-effect model was used to perform the meta-analysis. When $\mathrm{P}<0.05$ and $\mathrm{I}^{2}>50 \%$, it was considered that the trials had significant heterogeneity (16). The source of the heterogeneity was further analyzed. If there was no significant clinical heterogeneity, a secondary confirmatory analysis was performed with a random-effect model. Otherwise, descriptive analysis was performed. Where necessary, sensitivity analyses were performed to test the stability of identified outcomes.

\section{Results}

Literature search. The whole search process was as described in the flowchart in Fig. 1. The primary literature search identified 169 studies. Of these, 149 were excluded since they were not RCTs or were review studies. The remaining 20 studies were reviewed in full-length. Among them, 10 were excluded because they did not examine the target drugs, 2 were excluded since they were head-to-head comparisons, and 3 were excluded since the studies were based on the same patients and the same trial. Finally, 5 RCTs remained for inclusion in this meta-analysis. No patients in the involved studies had received previous systemic therapy. The 5 trials are randomized, multicenter, controlled and phase II or III trials. Among them, two assessed sorafenib $(17,18)$, one evaluated sunitinib (19), one evaluated pazopanib (20) and one evaluated axitinib (21). The methodological details relevant to bias and the treatment arms of the selected studies are presented in Table I.

Meta-analysis of the therapeutic efficiency of multikinase inhibitors

Progression free survival (PFS) and overall survival (OS). All studies reported PFS data and two of the studies reported OS data. Detailed information about the included trials is presented in Table II. The pooled HR of PFS is presented in Fig. 2. This shows that, compared with controls, multikinase inhibitors contributed to significantly longer PFS [hazard ratio $(\mathrm{HR})=0.58 ; 95 \%$ confidence interval $(\mathrm{CI}): 0.45-0.74$; $\mathrm{P}<0.0001]$. Of the two studies that reported OS data, one 
Table I. Summary of the included trials.

\begin{tabular}{|c|c|c|c|c|c|}
\hline Trial (ref.) & $\mathrm{N}$ & Intervention & Control & $\begin{array}{l}\text { Quality } \\
\text { components }\end{array}$ & $\begin{array}{l}\text { Quality } \\
\text { level }\end{array}$ \\
\hline Escudier 2007 (17) & 903 & $\begin{array}{l}\text { Sorafenib ( } 400 \mathrm{mg} \text {, twice daily, } \\
6 \text { week cycles) }\end{array}$ & Placebo & R; S and RPB; C; BR; F; ITT & B \\
\hline Escudier 2009 (18) & 189 & $\begin{array}{l}\text { Sorafenib (twice daily; } 400 \mathrm{mg} \text {, period } 1 \text {; } \\
600 \mathrm{mg} \text {, period 2) }\end{array}$ & IFN- $\alpha$ & R; S; C; BR; F; ITT & B \\
\hline Motzer 2009 (22) & 750 & $\begin{array}{l}\text { Sunitinib ( } 50 \mathrm{mg} \text { once daily, with a } \\
4 \text { weeks on and } 2 \text { weeks off schedule) }\end{array}$ & IFN- $\alpha$ & R; S and RPB; C; NB; F; ITT & B \\
\hline Sternberg 2010 (20) & 435 & Pazopanib (800 mg, once daily) & Placebo & R; S and RPB; C; DB; F; ITT & A \\
\hline Rini 2013 (21) & 112 & Axitinib (5 mg + $2 \mathrm{mg}$, twice daily) & Placebo & R; S and RPB; C; B R; F; ITT & B \\
\hline
\end{tabular}

All studies investigated patients with metastatic clear-cell renal cell carcinoma. R, randomized; S, stratification; RPB, random permuted blocks; BR, blind reviewer; DB, double blind; NB, non-blind; F, follow up; C, controlled; ITT, intent-to-treat.

Table II. Progression-free survival (PFS) and overall survival (OS) in the included trials.

\begin{tabular}{lccccccc}
\hline Trials (ref.) & $\mathrm{N}$ & Median PFS $^{\mathrm{a}}$ & $\mathrm{HR}(95 \% \mathrm{CI})$ & P-value & $\begin{array}{c}\text { Median OS } \\
\text { (months) }\end{array}$ & HR (95\% CI) & P-value \\
\hline Escudier 2007 (17) & 903 & 5.5 vs. 2.8 & $0.44(0.35-0.55)$ & 0.01 & 19.3 vs. 15.9 & $0.72(0.54-0.94)$ & 0.02 \\
Escudier 2009 (18) & 189 & 5.7 vs. 5.6 & $0.88(0.61-1.27)$ & 0.50 & N/A & - & - \\
Motzer 2009 (22) & 750 & 11.0 vs. 5.0 & $0.54(0.45-0.64)$ & 0.01 & 26.4 vs. 21.8 & $0.82(0.64-1.00)$ & NS \\
Sternberg 2010 (20) & 435 & 9.2 vs. 4.2 & $0.46(0.34-0.62)$ & 0.001 & N/A & - & - \\
Rini 2013 (21) & 112 & 14.5 vs. 15.7 & $0.85(0.54-1.35)$ & 0.24 & N/A & - & - \\
\hline
\end{tabular}

anvervention vs. control. HR, hazard ratio; CI, confidence interval; N/A, not available; NS, not significant.

study (17) reported a significantly longer OS for the multikinase inhibitor compared with the control (19.3 vs. 15.9 months, $\mathrm{P}=0.02$ ), while the other (22) did not find a significant difference (26.4 vs. 21.8 months, not significant).

$P D R$ and $O R R$. The number of patients with progressive disease was extracted to assess tumor progression. According to the meta-analysis, multikinase inhibitors are more effective in controlling tumor progression compared with placebo or IFN- $\alpha$ [relative risk $(\mathrm{RR})=0.67 ; 95 \%$ CI: $0.55-0.83 ; \mathrm{P}=0.0002$; Fig. 3). As to ORR, the effect of multikinase inhibitors was also superior to that of placebo or IFN- $\alpha(R R=2.93$; 95\% CI: 1.40-6.14; P=0.004; Fig. 4).

Adverse effects. The reported adverse effects associated with drug administration in the 5 trials were extracted and are summarized in Table III. Six types of adverse effect, including cardiac (hypertension), constitutional (fatigue) and gastrointestinal (diarrhea, anorexia and vomiting) effects and pain (abdominal) were reported. Only adverse effects reported in $\geq 4$ studies were pooled for RR evaluation. According to the meta-analysis, compared with IFN or placebo, multikinase inhibitors were associated with significantly higher rates of grade 3 or 4 hypertension ( $R R=6.00$; 95\% CI: 3.36-10.69; $\mathrm{P}<0.00001)$, diarrhea ( $\mathrm{R}=5.84 ; 95 \% \mathrm{CI}: 3.06-11.16$; $\mathrm{P}<0.00001$ ), nausea ( $\mathrm{RR}=2.30$; 95\% CI: $1.16-4.54 ; \mathrm{P}=0.02$ ), vomiting ( $\mathrm{RR}=1.84 ; 95 \% \mathrm{CI}: 1.00-3.41 ; \mathrm{P}=0.05)$ and hand -foot skin reaction $(\mathrm{RR}=11.78$; 95\% CI: 5.16-26.93; $\mathrm{P}<0.00001$;
Table III). However, the difference in fatigue between the multikinase inhibitor group and the IFN or placebo group was not significant ( $\mathrm{RR}=0.93$; 95\% CI: 0.69-1.25; $\mathrm{P}=0.61$; Table III).

\section{Discussion}

This meta-analysis aimed to identify the therapeutic effect and associated adverse events of the currently used multikinase inhibitors sorafenib, sunitinib, pazopanib and axitinib in advanced RCC. The results demonstrate that individual use of sorafenib, sunitinib, pazopanib and axitinib is more effective in terms of PDR and ORR. However, the results also show that multikinase inhibitors are associated with a higher risk of grade 3 or 4 adverse events for hypertension and gastrointestinal effects, including diarrhea, nausea and vomiting. Although much progress has been achieved in understanding the molecular mechanism of advanced RCC and in the development of targeting drugs, the overall efficiency of these therapies is far from satisfactory. Considering the poor physical condition of patients with advanced RCC, the appropriate selection of treatments and the proper management of adverse events are required to improve the quality of life of such patients.

Although the combination of bevacizumab and IFN may provide a higher ORR compared with either agent alone, the higher frequency or grade of adverse events may be unbearable for some patients (23). However, although the individual 


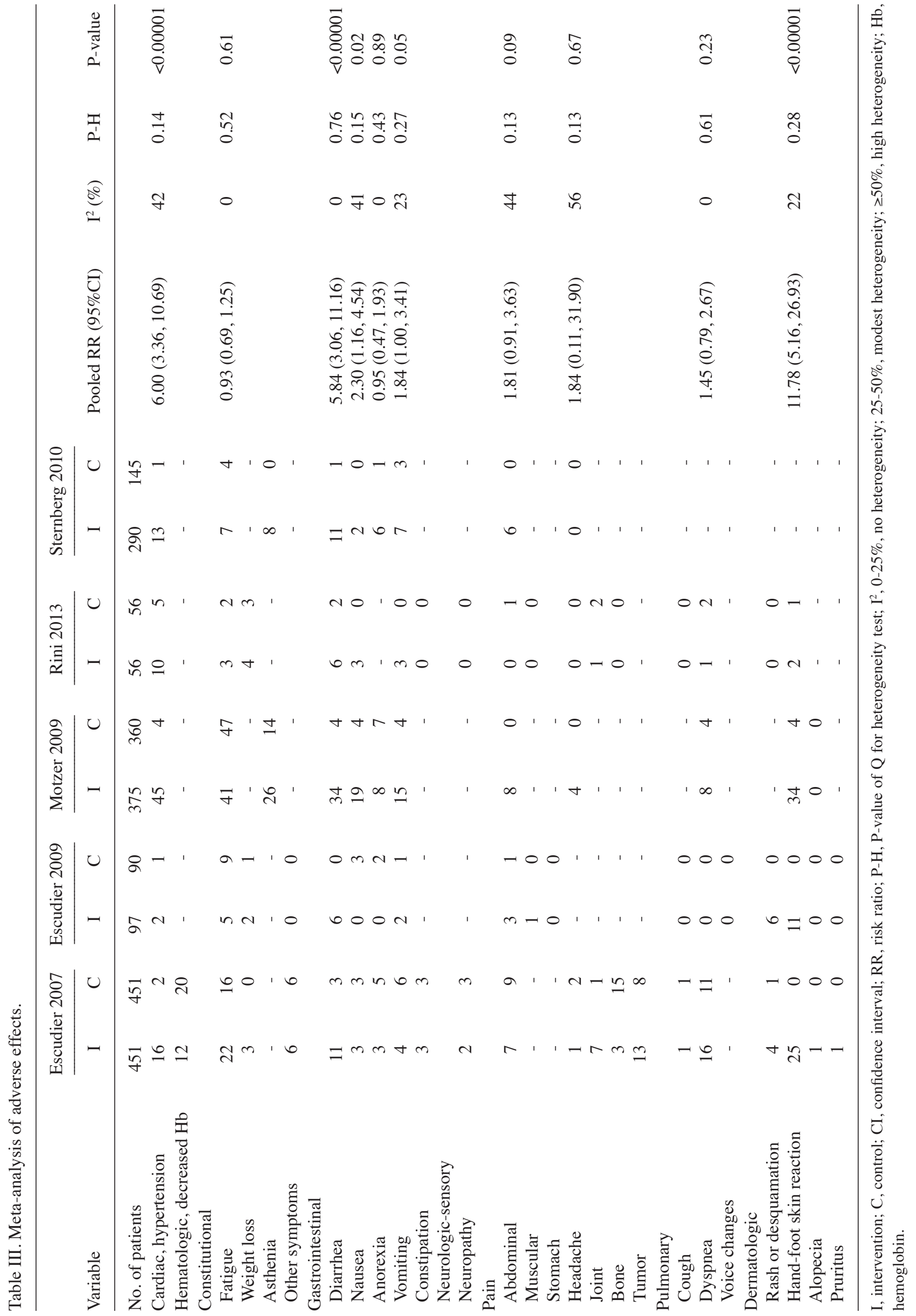




\begin{tabular}{|c|c|c|c|c|c|c|c|}
\hline Study or Subgroup & log[Hazard Ratio] & SE & Weight & $\begin{array}{c}\text { Hazard Ratio } \\
\text { IV, Random, 95\% CI }\end{array}$ & $\begin{array}{r}\text { Hazar } \\
\text { IV, Rando }\end{array}$ & $\begin{array}{l}\text { d Ratio } \\
\text { m, } 95 \% \mathrm{Cl}\end{array}$ & \\
\hline Escudier 2007 & -0.821 & 0.1168 & $23.1 \%$ & $0.44[0.35,0.55]$ & $=$ & & \\
\hline Escudier 2009 & -0.1278 & 0.187 & $17.5 \%$ & $0.88[0.61,1.27]$ & & & \\
\hline Motzer 2009 & -0.6162 & 0.093 & $24.9 \%$ & $0.54[0.45,0.65]$ & $\#$ & & \\
\hline Rini 2013 & -0.1625 & 0.2315 & $14.5 \%$ & $0.85[0.54,1.34]$ & - & & \\
\hline Sternberg 2010 & -0.7765 & 0.1542 & $20.0 \%$ & $0.46[0.34,0.62]$ & $\rightarrow$ & & \\
\hline Total $(95 \% \mathrm{Cl})$ & & & $100.0 \%$ & $0.58[0.45,0.74]$ & & & \\
\hline \multicolumn{8}{|c|}{ 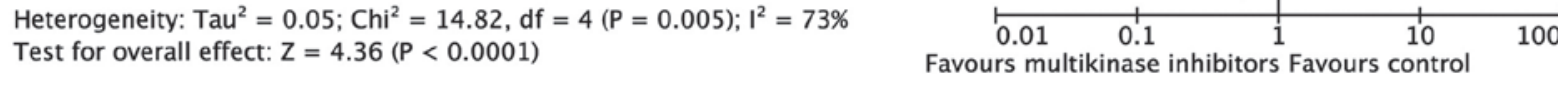 } \\
\hline
\end{tabular}

Figure 2. Pooled hazard ratio of progression-free survival. SE, standard error; IV, inverse variance; CI, confidence interval.

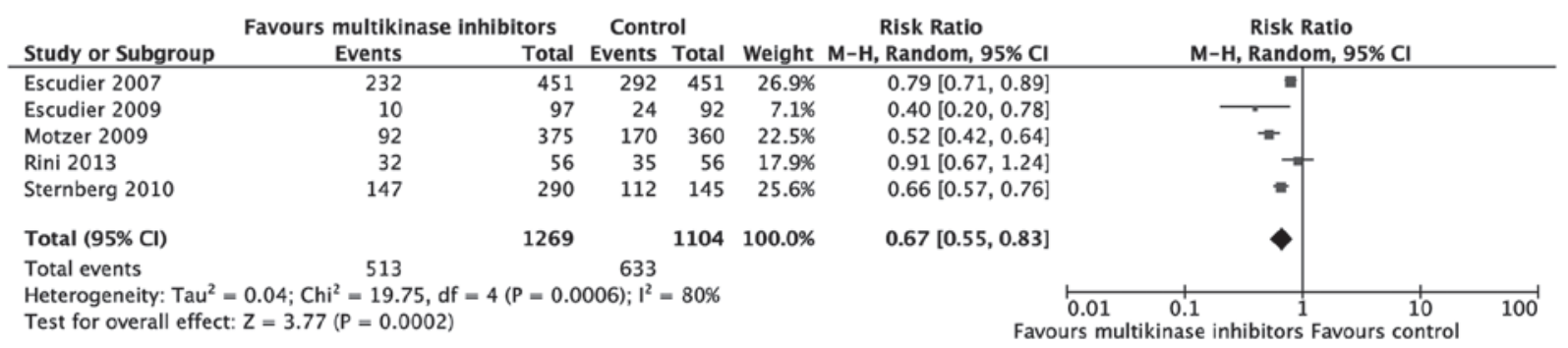

Figure 3. Meta-analysis of progressive disease rate. CI, confidence interval; M-H, Mantel-Haenszel.

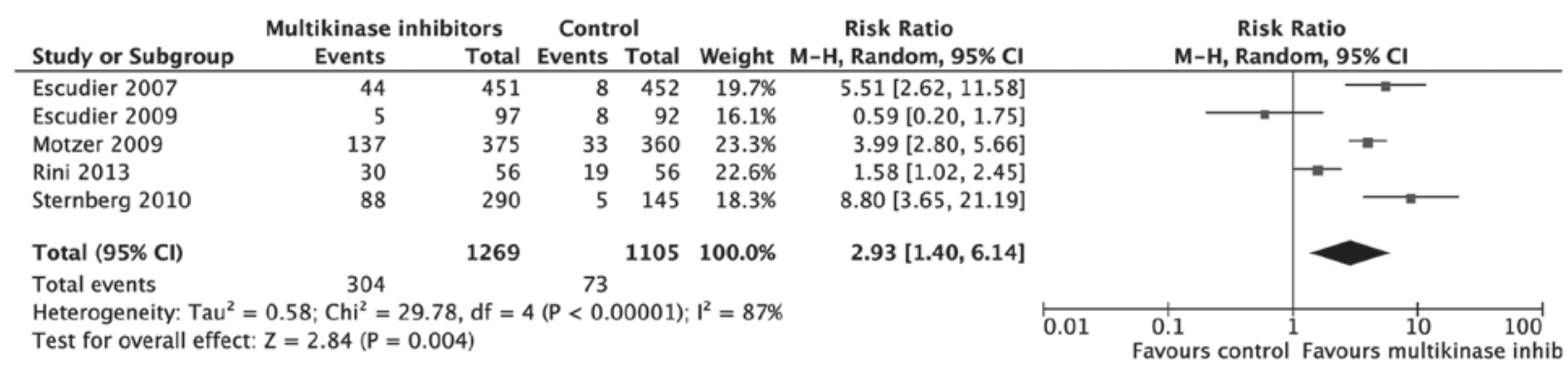

Figure 4. Meta-analysis of objective response rate. CI, confidence interval; M-H, Mantel-Haenszel.

use of sorafenib, sunitinib or temsirolimus is less toxic, the efficiency of these drugs in improving the ORR is limited (23). In clinical practice, the balance between therapeutic effect and associated adverse events should be carefully evaluated prior to the final selection of a treatment. Previous studies observed that patients suffered hypertension as early as the first day of taking sorafenib (24) and axitinib (25). Rini et al (26) observed that the median time of all-grade hypertension onset was independent of baseline antihypertensive use. However, the onset of grade 3 or 4 hypertension occurred significantly earlier in patients that received antihypertensive medications than in those that did not at baseline. Thus, patients receiving antihypertensive medication might be more biologically susceptible to earlier axitinib-induced hypertension (26). Therefore, it is recommended that clinicians consider collaborating with patients and other practitioners to manage treatment-induced hypertension actively. Prior to treatment, it is necessary for practitioners to assess the blood pressure status, cardiovascular risk factors and previous antihypertensive medication use of the patients. Based on this assessment, patients may be divided into normotensive, uncontrolled hypertensive, sub-optimally medication-controlled hypertensive or medication-controlled hypertensive groups (25). For patients with uncontrolled hypertension, short-acting antihypertensive agents could be provided. Following the initiation of multikinase treatment, short-acting antihypertensive agents may be switched to long-acting agents. For patients with sub-optimally controlled hypertension, treatment-induced hypertension could be controlled through increasing the dose of current medication or adding new medication. Pretreatment evaluation of risk factors of cardiovascular diseases, such as peripheral vascular disease, diabetes mellitus, cardiac conditions and renal disease is essential since patients with these risk factors may require close monitoring and the aggressive management of cardiovascular events during treatment (27). In addition, clinicians should to check whether patients have received hypertension-inducing agents such as hormones or steroids, which may complicate the management of axitinib-induced hypertension (28). It is recommended that during treatment with multikinase inhibitors, blood pressure should be monitored regularly in-clinic and at home. In order to gain a higher level of compliance of the patient to treatment, blood pressure assessment prior to treatment, including an assessment of the likelihood of hypertension developing during therapy and home monitoring could be provided. The clinician could support patients by training them and their family members in the proper use of the blood 
pressure-monitoring device, calibration of the device and recording and the reporting of measurements.

Gastrointestinal adverse events, including diarrhea, nausea and vomiting were commonly reported for these multikinase inhibitors. Although these events might not lead to treatment discontinuation and can be properly managed by pharmacological intervention and dietary modifications, in elderly patients, these events might lead to serious dehydration if they are not well controlled (29). Previous studies also reported that treatment-related diarrhea can be persistent for the duration of multikinase therapy and mild-to-moderate diarrhea can reduce the mobility and independence of patients, impairing quality of life (30-32). Therefore, it is recommended that clinical guidelines for the management of cancer treatment-related gastrointestinal adverse events should be followed (33). The major limitation of this study is the small number of studies involved. This small selection of studies was not adequate to perform patient subgroup analyses.

In conclusion, multikinase inhibitors can significantly control disease progress and improve the ORR. However, they are also associated with a higher risk of grade 3 and 4 hypertension and gastrointestinal events. Proper management of these events is necessary to improve quality of life.

\section{References}

1. Decastro GJ and McKiernan JM: Epidemiology, clinical staging and presentation of renal cell carcinoma. Urol Clin North Am 35: 581-592, 2008

2. Jemal A, Siegel R, Xu J and Ward E: Cancer statistics, 2010. CA Cancer J Clin 60: 277-300, 2010.

3. Garcia JA, Cowey CL and Godley PA: Renal cell carcinoma Curr Opin Oncol 21: 266-271, 2009.

4. Motzer RJ, Bander NH and Nanus DM: Renal-cell carcinoma. N Engl J Med 335: 865-875, 1996.

5. van Spronsen DJ, de Weijer KJ, Mulders PF and De Mulder PH: Novel treatment strategies in clear-cell metastatic renal cell carcinoma. Anticancer Drugs 16: 709-717, 2005.

6. Van Veldhuizen PJ, Hussey M, Lara PN Jr, et al: A phase II study of gemcitabine and capecitabine in patients with advanced renal cell cancer: Southwest Oncology Group Study S0312. Am J Clin Oncol, 2009.

7. Escudier B, Eisen T, Stadler WM, et al; TARGET Study Group: Sorafenib in advanced clear-cell renal-cell carcinoma. N Engl J Med 356: 125-134, 2007.

8. Motzer RJ, Michaelson MD, Rosenberg J, et al: Sunitinib efficacy against advanced renal cell carcinoma. J Urol 178: 1883-1887, 2007.

9. Xu CF, Bing NX, Ball HA, et al: Pazopanib efficacy in renal cell carcinoma: evidence for predictive genetic markers in angiogenesis-related and exposure-related genes. J Clin Oncol 29: 2557-2564, 2011.

10. Cella D, Escudier B, Rini B, et al: Patient-reported outcomes for axitinib vs sorafenib in metastatic renal cell carcinoma: phase III (AXIS) trial. Br J Cancer 108: 1571-1578, 2013.

11. Escudier B, Pluzanska A, Koralewski P, et al; AVOREN Trial Investigators: Bevacizumab plus interferon alfa-2a for treatment of metastatic renal cell carcinoma: a randomised, double-blind phase III trial. Lancet 370: 2103-2111, 2007.
12. Hudes G, Carducci M, Tomczak P, et al: Temsirolimus, interferon alfa, or both for advanced renal-cell carcinoma. N Engl J Med 356: 2271-2281, 2007.

13. Motzer RJ, Escudier B, Oudard S, et al: Efficacy of everolimus in advanced renal cell carcinoma: a double-blind, randomised, placebo-controlled phase III trial. Lancet 372: 449-456, 2008.

14. Kim HL, Belldegrun AS, Freitas DG, et al: Paraneoplastic signs and symptoms of renal cell carcinoma: implications for prognosis. J Urol 170: 1742-1746, 2003.

15. Higgins JPT and Green S (eds): Cochrane Handbook for Systematic Reviews of Interventions. John Wiley \& Sons, Inc., Chichester, UK, 2008.

16. Higgins JP, Thompson SG, Deeks JJ and Altman DG: Measuring inconsistency in meta-analyses. BMJ 327: 557-560, 2003.

17. Escudier B, Eisen T, Stadler WM, et al; TARGET Study Group: Sorafenib in advanced clear-cell renal-cell carcinoma. N Engl J Med 356: 125-134, 2007.

18. Escudier B, Szczylik C, Hutson TE, et al: Randomized phase II trial of first-line treatment with sorafenib versus interferon Alfa-2a in patients with metastatic renal cell carcinoma. J Clin Oncol 27: 1280-1289, 2009.

19. Motzer RJ, Hutson TE, Tomczak P, et al: Sunitinib versus interferon alfa in metastatic renal-cell carcinoma. $\mathrm{N}$ Engl J Med 356: 115-124, 2007.

20. Sternberg CN, Davis ID, Mardiak J, et al: Pazopanib in locally advanced or metastatic renal cell carcinoma: results of a randomized phase III trial. J Clin Oncol 28: 1061-1068, 2010.

21. Rini BI, Melichar B, Ueda T, et al: Axitinib with or without dose titration for first-line metastatic renal-cell carcinoma: a randomised double-blind phase 2 trial. Lancet Oncol 14: 1233-1242, 2013.

22. Motzer RJ, Hutson TE, Tomczak P, et al: Overall survival and updated results for sunitinib compared with interferon alfa in patients with metastatic renal cell carcinoma. J Clin Oncol 27: 3584-3590, 2009.

23. Escudier B, Albiges L and Sonpavde G: Optimal management of metastatic renal cell carcinoma: Current status. Drugs 73: 427-438, 2013.

24. Maitland ML, Kasza KE, Karrison T, et al: Ambulatory monitoring detects sorafenib-induced blood pressure elevations on the first day of treatment. Clin Cancer Res 15: 6250-6257, 2009.

25. Rixe O, Bukowski RM, Michaelson MD, et al: Axitinib treatment in patients with cytokine-refractory metastatic renal-cell cancer: a phase II study. Lancet Oncol 8: 975-984, 2007.

26. Rini BI, Quinn DI, Baum M, et al: Hypertension among patients with renal cell carcinoma receiving axitinib or sorafenib: analysis from the randomized phase III AXIS trial. Target Oncol, 2014.

27. Edmonds K, Hull D, Spencer-Shaw A, et al: Strategies for assessing and managing the adverse events of sorafenib and other targeted therapies in the treatment of renal cell and hepatocellular carcinoma: recommendations from a European nursing task group. Eur J Oncol Nurs 16: 172-184, 2012.

28. Hutson TE, Figlin RA, Kuhn JG and Motzer RJ: Targeted therapies for metastatic renal cell carcinoma: an overview of toxicity and dosing strategies. Oncologist 13: 1084-1096, 2008.

29. O'Brien BE, Kaklamani VG and Benson AB III: The assessment and management of cancer treatment-related diarrhea. Clin Colorectal Cancer 4: 375-381; discussion 382-373, 2005.

30. Bellmunt J, Eisen T, Fishman M and Quinn D: Experience with sorafenib and adverse event management. Crit Rev Oncol Hematol 78: 24-32, 2011.

31. La Vine DB, Coleman TA, Davis CH, Carbonell CE and Davis WB: Frequent dose interruptions are required for patients receiving oral kinase inhibitor therapy for advanced renal cell carcinoma. Am J Clin Oncol 33: 217-220, 2010.

32. Dutcher JP, Tannir N, Bellmunt J and Escudier B: Experience with sorafenib and the elderly patient. Med Oncol 27: 1359-1370, 2010.

33. Benson AB III, Ajani JA, Catalano RB, et al: Recommended guidelines for the treatment of cancer treatment-induced diarrhea. J Clin Oncol 22: 2918-2926, 2004. 